\title{
In vivo evaluation of percutaneous carbon dioxide treatment for improving intratumoral hypoxia using ${ }^{18}$ F-fluoromisonidazole PET-CT
}

\author{
KOJI MARUYAMA ${ }^{1}$, TAKUYA OKADA ${ }^{1}$, TAKESHI UEHA ${ }^{2}$, KAYAKO ISOHASHI $^{3}$, \\ HAYATO IKEDA $^{3}$, YASUKAZU KANAI ${ }^{3}$, KOJI SASAKI ${ }^{1}$, TOMOYUKI GENTSU ${ }^{1}$, \\ EISUKE UESHIMA $^{1}$, KEITARO SOFUE ${ }^{1}$, MUNENOBU NOGAMI ${ }^{1}$, MASATO YAMAGUCHI $^{1}$, \\ KOJI SUGIMOTO ${ }^{1}$, YOSHITADA SAKAI ${ }^{2}$, JUN HATAZAWA ${ }^{3}$ and TAKAMICHI MURAKAMI ${ }^{1}$ \\ ${ }^{1}$ Department of Radiology, Kobe University Graduate School of Medicine; ${ }^{2}$ Division of Rehabilitation Medicine, \\ Kobe University Graduate School of Medicine, Kobe, Hyogo 650-0017; ${ }^{3}$ Department of Tracer Kinetics and \\ Nuclear Medicine, Osaka University Graduate School of Medicine, Suita, Osaka 565-0871, Japan
}

Received July 31, 2020; Accepted December 22, 2020

DOI: $10.3892 / \mathrm{ol} .2021 .12468$

\begin{abstract}
Carbon dioxide $\left(\mathrm{CO}_{2}\right)$ treatment is reported to have an antitumor effect owing to the improvement in intratumoral hypoxia. Previous studies were based on histological analysis alone. In the present study, the improvement in intratumoral hypoxia by percutaneous $\mathrm{CO}_{2}$ treatment in vivo was determined using ${ }^{18} \mathrm{~F}$-fluoromisonidazole positron emission tomographycomputed tomography $\left({ }^{18} \mathrm{~F}-\mathrm{FMISO}\right.$ PET-CT) images. Twelve Japanese nude mice underwent implantation of LM8 tumor cells in the dorsal subcutaneous area 2 weeks before percutaneous $\mathrm{CO}_{2}$ treatment and ${ }^{18} \mathrm{~F}$-FMISO PET-CT scans. Immediately after intravenous injection of ${ }^{18} \mathrm{~F}-\mathrm{FMISO}, \mathrm{CO}_{2}$ and room air were administered transcutaneously in the $\mathrm{CO}_{2}$-treated group $(n=6)$ and a control group $(n=6)$, respectively; each treatment was performed for 10 minutes. PET-CT was performed $2 \mathrm{~h}$ after administration of ${ }^{18} \mathrm{~F}$-FMISO ${ }^{18} \mathrm{~F}$-FMISO tumor uptake was quantitatively evaluated using the maximum standardized uptake value ( $\mathrm{SUV}_{\max }$ ), tumor-to-liver ratio (TLR), tumor-tomuscle ratio (TMR), metabolic tumor volume (MTV) and total lesion glycolysis (TLG). Mean \pm standard error of the mean (SEM) of the tumor volume was not significantly different between the two groups $\left(\mathrm{CO}_{2}\right.$-treated group, $1.178 \pm 0.450 \mathrm{~cm}^{3}$; control group, $1.368 \pm 0.295 \mathrm{~cm} 3 ; \mathrm{P}=0.485)$. Mean $\pm \mathrm{SEM}$ of $\mathrm{SUV}_{\max }$, TLR, MTV (cm3) and TLG were significantly lower in the $\mathrm{CO}_{2}$-treated group compared with the control group $(0.880 \pm 0.095$ vs. $1.253 \pm 0.071, \mathrm{P}=0.015 ; 1.063 \pm 0.147361$ vs
\end{abstract}

Correspondence to: Dr Takuya Okada, Department of Radiology, Kobe University Graduate School of Medicine, 7-5-2 Kusunoki-cho, Chuo-ku, Kobe, Hyogo 650-0017, Japan

E-mail: okabone@gmail.com

Key words: carbon dioxide, intratumoral hypoxia, in vivo, LM8 tumor, ${ }^{18} \mathrm{~F}$-fluoromisonidazole positron emission tomographycomputed tomography
$1.455 \pm 0.078, \mathrm{P}=0.041 ; 0.353 \pm 0.139$ vs. $1.569 \pm 0.438, \mathrm{P}=0.015$; $0.182 \pm 0.070$ vs. $1.028 \pm 0.338, \mathrm{P}=0.015)$, respectively. TMR was not significantly different between the two groups $(4.520 \pm 0.503$ vs. 5.504 $\pm 0.310 ; \mathrm{P}=0.240)$. In conclusion, ${ }^{18} \mathrm{~F}-\mathrm{FMISO} \mathrm{PET}$ revealed that percutaneous $\mathrm{CO}_{2}$ treatment improved intratumoral hypoxia in vivo. This technique enables assessment of the therapeutic effect in $\mathrm{CO}_{2}$ treatment by imaging, and may contribute to its clinical application.

\section{Introduction}

Intratumoral hypoxia can show potential therapeutic resistance owing to its adverse impact on the effectiveness of chemotherapy and radiotherapy. The rapid growth of solid tumors changes the cellular microenvironment, leading to inadequate oxygen supply, which results in intratumoral hypoxia $(1,2)$.

Carbon dioxide $\left(\mathrm{CO}_{2}\right)$ treatment is reported to have an antitumor effect due to the improvement in intratumoral hypoxia. The beneficial effects of $\mathrm{CO}_{2}$ treatment include an increase in blood flow and microcirculation, neocapillary formation depending on nitric oxide, and partial increase in oxygen pressure in a confined space, known as the Bohr effect (3), which has been applied for a treatment for skin disorder (4-6). Recently, transcutaneous administration or intra-arterial infusion of $\mathrm{CO}_{2}$ was reported to have an antitumor effect in animal experiments (7-11). In previous studies, improvement in intratumoral hypoxia by $\mathrm{CO}_{2}$ treatment was pathologically proven by decreased hypoxia-inducible factor (HIF-1) $\alpha$ expression and increased carbonic anhydrase IX expression in the excised specimens $(9,11)$. However, no study has demonstrated an improvement in intratumoral hypoxia by $\mathrm{CO}_{2}$ treatment in living organisms. A noninvasive method of evaluation is required for future clinical applications of $\mathrm{CO}_{2}$ treatment.

PET-CT using radiolabeled hypoxia-avid compounds is a noninvasive method that can visualize hypoxia with several types of hypoxic tracers. ${ }^{18} \mathrm{~F}$-fluoromisonidazole (FMISO), a labeled 2-nitroimidazole compound, is one of the most widely 
used hypoxic tracers $(12) .{ }^{18} \mathrm{~F}$-FMISO PET-CT is a wellestablished method of hypoxic imaging and has been used not only in animal research but also in clinical practice (13).

This study aimed to determine the improvement in intratumoral hypoxia by percutaneous $\mathrm{CO}_{2}$ treatment in vivo using ${ }^{18}$ F-FMISO PET images.

\section{Materials and methods}

Animal preparation and implantation of LM8 osteosarcoma cells. This study was approved by the Institutional Animal Care and Use Committee (permit no. P160903 and 28-043-000) and was performed in accordance with the Kobe University Animal Experimentation Regulations and the Osaka University Regulations on Animal Experiments.

A murine osteosarcoma cell line (LM8; cat. no. RCB1450; Riken BRC Cell Bank) was used in the present study. The cells were cultured in a growth medium composed of Dulbecco's modified Eagle's medium (DMEM; SigmaAldrich; Merck KGaA) added to $10 \%$ (v/v) fetal bovine serum (FBS; Sigma-Aldrich; Merck KGaA) and $100 \mathrm{U} / \mathrm{ml}$ penicillin/streptomycin solution (Sigma-Aldrich; Merck $\mathrm{KGaA}$ ). The cells were maintained under $37^{\circ} \mathrm{C}$ in a humidified $5 \% \mathrm{CO}_{2}$ atmosphere.

Male BALB/c nude mice [age, 5 weeks; body weight (mean \pm standard deviation), $20.7 \pm 0.8 \mathrm{~g}]$ were obtained from CLEA Japan, Inc. The animals were housed under pathogenfree conditions in accordance with institutional principles. After one week, LM8 cells (1.0x10 $0^{6}$ cells in $500 \mu$ l PBS) were implanted into the dorsal subcutaneous area of the mice as previously described (Fig. 1) (14).

Animal studies. Transcutaneous administration of $\mathrm{CO}_{2}$ and ${ }^{18} \mathrm{~F}$-FMISO PET-CT scans were scheduled at 2 weeks after LM8 cell implantation. Mice with tumor xenografts $(n=12)$ were randomly assigned into two groups: $\mathrm{CO}_{2}$-treated group $(n=6)$ and an untreated group $(n=6)$. Transcutaneous administration of $\mathrm{CO}_{2}$ was performed in the $\mathrm{CO}_{2}$ treatment group immediately after intravenous injection of ${ }^{18} \mathrm{~F}$-FMISO, while room air was administered in the untreated group. Each treatment was performed for $10 \mathrm{~min}$. PET-CT was performed $2 \mathrm{~h}$ after intravenous administration of ${ }^{18} \mathrm{~F}$-FMISO. After the treatment and imaging, all mice were euthanized by intraperitoneal injection of $200 \mathrm{mg} / \mathrm{kg}$ sodium pentobarbital (Somnopenthyl, $64.8 \mathrm{mg} / \mathrm{ml}$; Kyoritsu Seiyaku).

Transcutaneous $\mathrm{CO}_{2}$ application. Transcutaneous administration of $\mathrm{CO}_{2}$ was performed as described previously $(8,9,15)$. All mice were anesthetized with $2 \%$ isoflurane in room air. The area of skin surrounding the implanted tumor was covered with $\mathrm{CO}_{2}$ hydrogel. As this part was contained, the lower part of the mouse was sealed with a polyethylene bag, into which $100 \% \mathrm{CO}_{2}$ was then administered. The mice in the untreated group underwent the same procedure except that $\mathrm{CO}_{2}$ was replaced with room air.

${ }^{18} \mathrm{~F}$-FMISO PET-CT scan. ${ }^{18} \mathrm{~F}$-FMISO was prepared using the same method as previously mentioned (16). ${ }^{18} \mathrm{~F}-\mathrm{HF}$ was produced by an in-house cyclotron (HM-12S; Sumitomo Heavy Industry) and ${ }^{18} \mathrm{~F}$-FMISO was prepared with a UG-M1 synthesizer module using 3-(2-nitroimidazo-1-yl)-2-Otetrahydropyranyl-1-O-toluenesulfonyl-propanediol as a labeling precursor. ${ }^{18}$ F-FMISO PET-CT was subsequently performed $(16,17)$.

All the mice were anesthetized with $2 \%$ isoflurane in room air, and a Terumo $29-\mathrm{G}$ needle-embedded insulin syringe was inserted into the tail vein for ${ }^{18} \mathrm{~F}$-FMISO injection. The mean \pm standard error of the mean (SEM) for the injected dose of ${ }^{18} \mathrm{~F}$-FMISO was $19.38 \pm 0.82 \mathrm{MBq}$. In a small-animal PET system (Inveon PET-CT system; Siemens Medical Solutions), the animals were placed in a feet-first and supine position under anesthesia with $2 \%$ isoflurane at room air. Static emission scans were performed at $2 \mathrm{~h}$ post-injection, with an emission scan of $10 \mathrm{~min}$, and $\mathrm{CT}$ acquisition was performed immediately before PET acquisition.

All PET-CT images were reconstructed with 3-dimensional ordered-subset expectation maximization followed by maximum a posteriori (OSEM3D-MAP) (16 subsets, 2 OSEM3D and 18 MAP iterations) and CT-based attenuation correction. The image matrix was $128 \times 128 \times 159$, which produced a voxel size of $0.776 \times 0.776 \times 0.796 \mathrm{~mm}$.

Image analysis. All images were analyzed on a clinical Osirix MD workstation (Pixmeo) using Metavol software (http://www.metavol.org/) (18).

The tumor volume was calculated based on the volume measured on the CT images. ${ }^{18} \mathrm{~F}$-FMISO uptake by the tumor was evaluated quantitatively using the maximum standardized uptake value ( $\mathrm{SUV}_{\max }$ ), tumor-to-liver ration (TLR), tumor-tomuscle ration (TMR), metabolic tumor volume (MTV) and total lesion glycolysis (TLG). A polygonal volume of interest (VOI), which included the entire lesion in the axial, sagittal, and coronal planes, was also examined. The parameters were defined as the following: $\mathrm{SUV}_{\max }$, the $\mathrm{SUV}$ of the single highest voxel in the tumor; TMR, the ratio of $\mathrm{SUV}_{\max }$ of the tumor to the mean SUV of the gluteal muscle; TLR, the $\mathrm{SUV}_{\max }$ of the tumor/the mean SUV of the liver; MTV, the volume of the tumor that exhibited FDG uptake; and TLG, the mean SUV of the tumor x MTV. To determine the MTV, images of the tumor were segmented using a fixed-threshold (SUV, 0.4), referring to the past report (19). The threshold value was determined by measuring $40 \%$ of the mean $\mathrm{SUV}_{\max }$ of all tumors (mean \pm SEM, 1.07 \pm 0.28 ).

Statistical analysis. All parameters were compared between the two groups. Data are expressed as the mean \pm SEM, unless indicated otherwise. The difference between the two groups was evaluated using the Mann-Whitney U-test. All statistical analyses were performed using EZR (Saitama Medical Center, Jichi Medical University), a graphical user interface for R (The $\mathrm{R}$ Foundation for Statistical Computing; version 2.13.0). It is a modified version of $\mathrm{R}$ commander (version 1.6-3), designed with additional statistical functions used more frequently in biostatistics (20). $\mathrm{P}<0.05$ was considered to indicate a statistically significant difference.

\section{Results}

A total of 12 mice [age, 8 weeks; body weight (mean \pm SEM), $23.86 \pm 0.39 \mathrm{~g}$ ] were randomly assigned into two groups: The $\mathrm{CO}_{2}$-treated group $(\mathrm{n}=6)$ and the control group $(\mathrm{n}=6)$. 


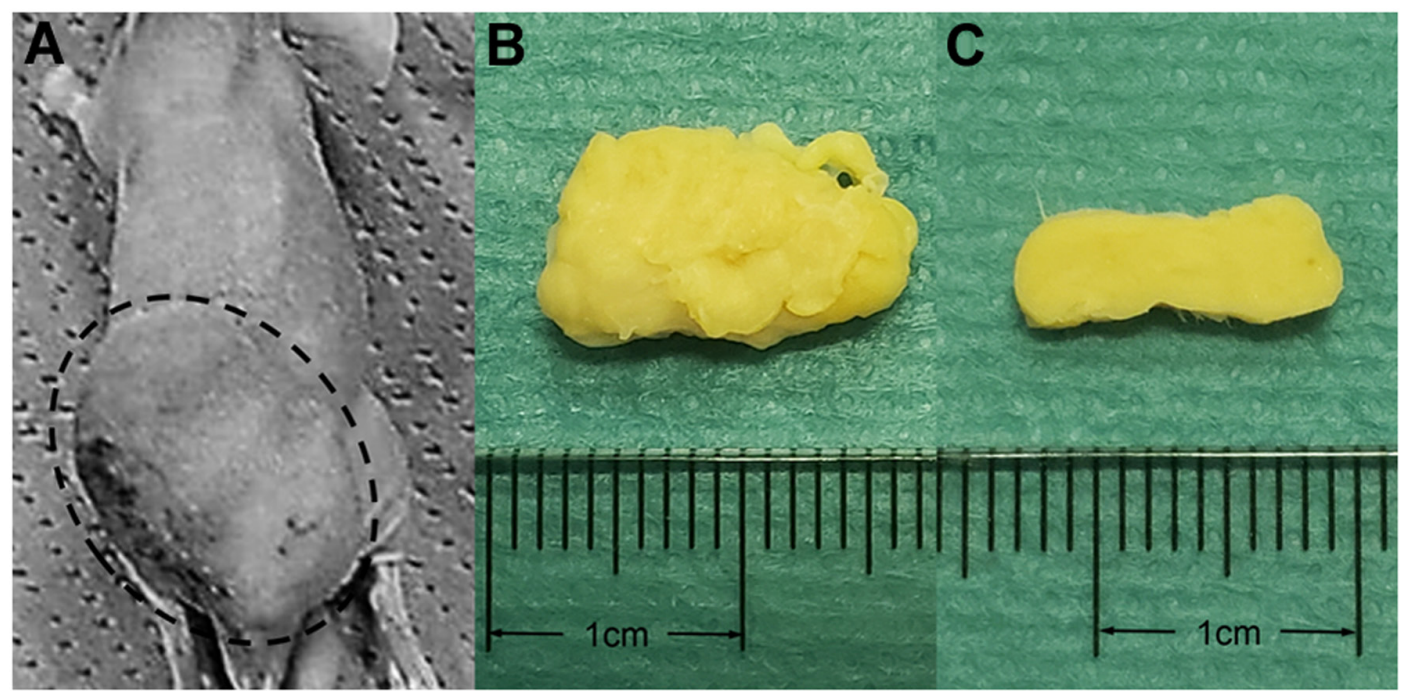

Figure 1. Images of the implanted tumors. (A) A mouse with tumor 2 weeks after LM8 cell implantation. (B) Excised tumor and (C) its cross section after formalin fixation. The tumor volume, the short and long dimensions at the endpoint were $0.361 \mathrm{~cm}^{3}, 0.89$ and $1.21 \mathrm{~cm}$, respectively. No macroscopically apparent necrosis was noted in the excised tumor.

Table I. Characteristics of the animals in the present study.

\begin{tabular}{lccr}
\hline Characteristics & $\mathrm{CO}_{2}$-treated group, $\mathrm{n}=6$ & Control group, $\mathrm{n}=6$ & P-value \\
\hline Body weight, $\mathrm{g}$ & & & 0.818 \\
Mean \pm SEM & $23.98 \pm 0.55$ & $23.73 \pm 0.59$ & \\
Range & $22.62-26.06$ & $21.92-25.79$ & 0.937 \\
Injected dose of ${ }^{18} \mathrm{~F}-\mathrm{FMISO}, \mathrm{MBq}$ & & $19.08 \pm 1.39$ & \\
Mean \pm SEM & $19.69 \pm 1.00$ & $13.03-22.55$ & 0.485 \\
Range & $16.84-22.91$ & & \\
Tumor volume, $\mathrm{cm}^{3}$ & & $1.368 \pm 0.295$ & \\
Mean \pm SEM & $1.178 \pm 0.450$ & $0.433-2.151$ & \\
Range & $0.361-3.148$ & & \\
\hline
\end{tabular}

SEM, standard error of the mean; FMISO, fluoromisonidazole.

The animal characteristics are summarized in Table I. There were no significant differences between the two groups with respect to weight, injected dose of ${ }^{18} \mathrm{~F}-\mathrm{FMISO}$, and the tumor volume.

Fig. 2 shows the representative cases in each group. The mean value of $\mathrm{SUV}_{\max }$ of the tumor, TMR, TLR, MTV and TLG are summarized in Table II. SUV $\mathrm{max}_{\max }$ of the tumor, TLR, MTV and TLG in the $\mathrm{CO}_{2}$-treated group were significantly lower compared with those in the control group. No significant difference in TMR was observed between the two groups (Fig. 3).

\section{Discussion}

This study demonstrated the improvement in intratumoral hypoxia by percutaneous $\mathrm{CO}_{2}$ treatment; this was later ascertained using ${ }^{18} \mathrm{~F}$-FMISO PET images in the living state. As most tumors cannot be diagnosed clinically, ${ }^{18} \mathrm{~F}$-FMISO PET may contribute to the clinical use of $\mathrm{CO}_{2}$ treatment by measuring the response of various tumors to $\mathrm{CO}_{2}$ administration.

The reoxygenation and anticancer effects of $\mathrm{CO}_{2}$ therapy were previously reported (9). Transcutaneous $\mathrm{CO}_{2}$ application significantly decreased tumor growth in mice implanted with LM8 cells, the same experimental platform used in the present study. Apoptotic activity increased and intratumoral hypoxia improved with decreased expression of HIF-1 $\alpha$. Pimonidazole, a 2-nitroimidazole that is activated specifically in hypoxic cells, was used to assess intratumoral hypoxia directly. HIF-1 $\alpha$ accumulates under hypoxic conditions; along with its downstream genes such as matrix metalloproteinases, it confers poor prognosis in many types of tumors (9). The previous studies were based on histological analysis alone; this study suggests that the hypoxic condition actually improves in the living state.

${ }^{18} \mathrm{~F}-\mathrm{FMISO}$ has high affinity for hypoxic cells with functional nitro reductase enzymes (21). The rate of ${ }^{18} \mathrm{~F}$-FMISO binding increases when the partial pressure of oxygen is less 


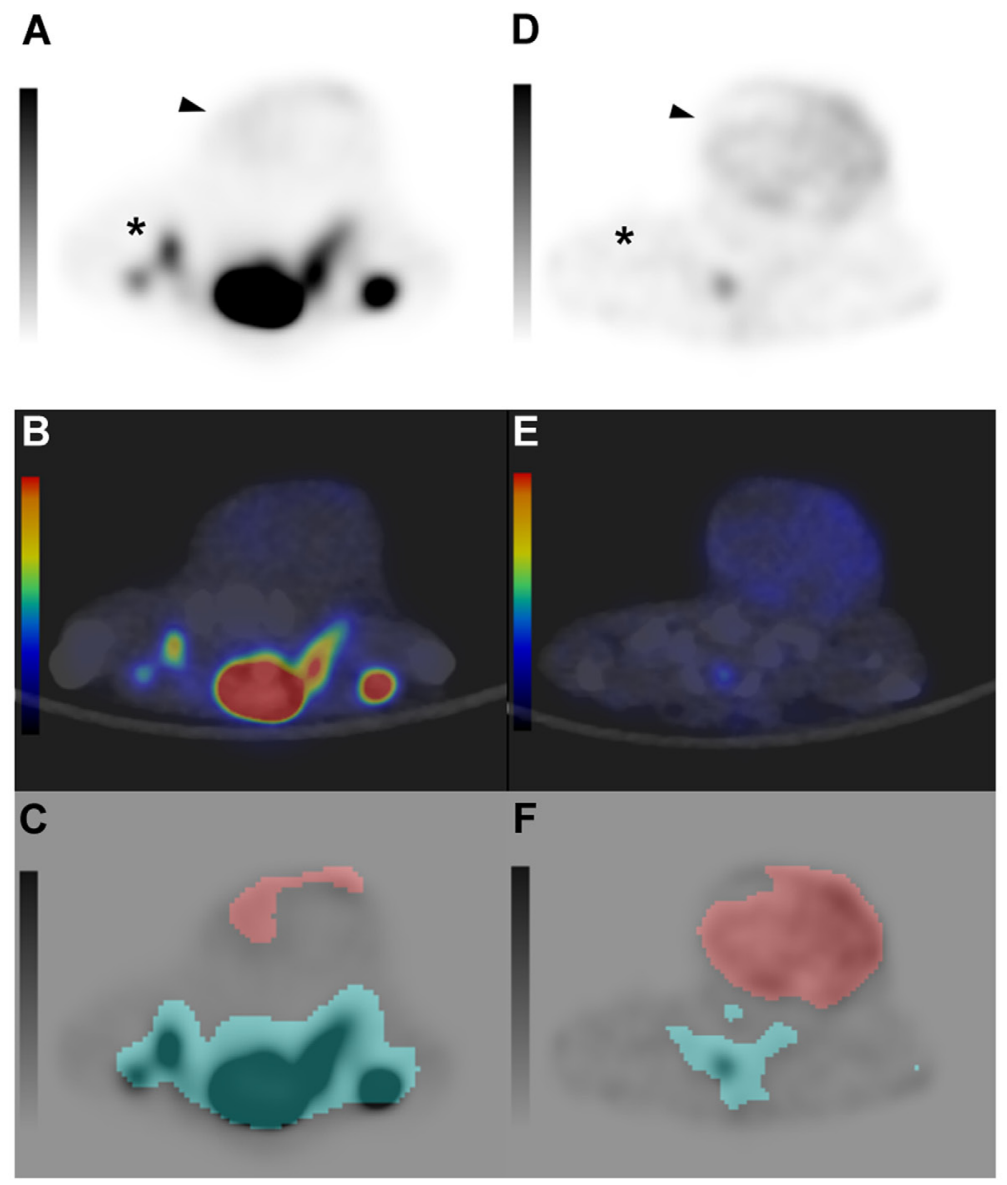

Figure 2. Axial images of ${ }^{18} \mathrm{~F}$-FMISO PET-CT. (A-C) Axial images of ${ }^{18} \mathrm{~F}-\mathrm{FMISO}$ PET, PET-CT fusion, and the MTV measurement in the $\mathrm{CO}_{2}$-treated group and (D-F) control group. ${ }^{18} \mathrm{~F}$-FMISO PET and PET-CT fusion image using a color map shows ${ }^{18} \mathrm{~F}$-FMISO uptake in the tumor located in the gluteal region of mice (arrowhead). Red area on the MTV measurement image indicates a tumoral area with a SUV exceeding 0.4. ${ }^{18} \mathrm{~F}$-FMISO uptake in the $\mathrm{CO}_{2}$-treated group $\left(\mathrm{SUV}_{\text {max }}, 0.669 ; \mathrm{MTV}, 0.56 \mathrm{~cm}^{3}\right)$ is lower compared with the control group $\left(\mathrm{SUV}_{\max }, 1.532 ; \mathrm{MTV}, 3.111 \mathrm{~cm}^{3}\right)$. The dorsal uptake of the tumor (*) is physiological. ${ }^{18} \mathrm{~F}$-FMISO, ${ }^{18} \mathrm{~F}$ - fluoromisonidazole; PET-CT positron emission tomography/computed tomography; MTV, metabolic tumor volume; SUV, standardized uptake value; $\mathrm{SUV}_{\max }$, maximum SUV.

Table II. Mean SUV $\max$, TMR, TLR, MTV and TLG values in the present study.

\begin{tabular}{lcc}
\hline Variables & $\mathrm{CO}_{2}$-treated group, $\mathrm{n}=6$ & Control group, $\mathrm{n}=6$ \\
\hline $\mathrm{SUV}_{\text {max }}$ & $0.880 \pm 0.095$ & $1.253 \pm 0.071$ \\
Mean \pm SEM & $0.587-1.241$ & $1.076-1.532$ \\
Range & & \\
TLR & $1.063 \pm 0.147$ & $1.455 \pm 0.078$ \\
Mean \pm SEM & $0.533-1.521$ & $1.165-1.654$ \\
Range & & \\
TMR & $4.520 \pm 0.503$ & $5.504 \pm 0.310$ \\
Mean \pm SEM & $2.836-6.144$ & $4.816-6.656$ \\
Range & & $1.569 \pm 0.438$ \\
MTV, cm ${ }^{3}$ & $0.353 \pm 0.139$ & $0.391-3.111$ \\
Mean \pm SEM & $0.073-0.934$ & 0.041 \\
Range & & $1.028 \pm 0.338$ \\
TLG & $0.182 \pm 0.070$ & $0.220-2.318$ \\
Mean \pm SEM & $0.022-0.483$ & 0.015 \\
Range & & \\
\hline
\end{tabular}

SEM, standard error of the mean; $\mathrm{SUV}_{\max }$, maximum standardized uptake value; TLR, tumor-to-liver ratio; TMR, tumor-to-muscle ratio; MTV, metabolic tumor volume; TLG, total lesion glycolysis. 

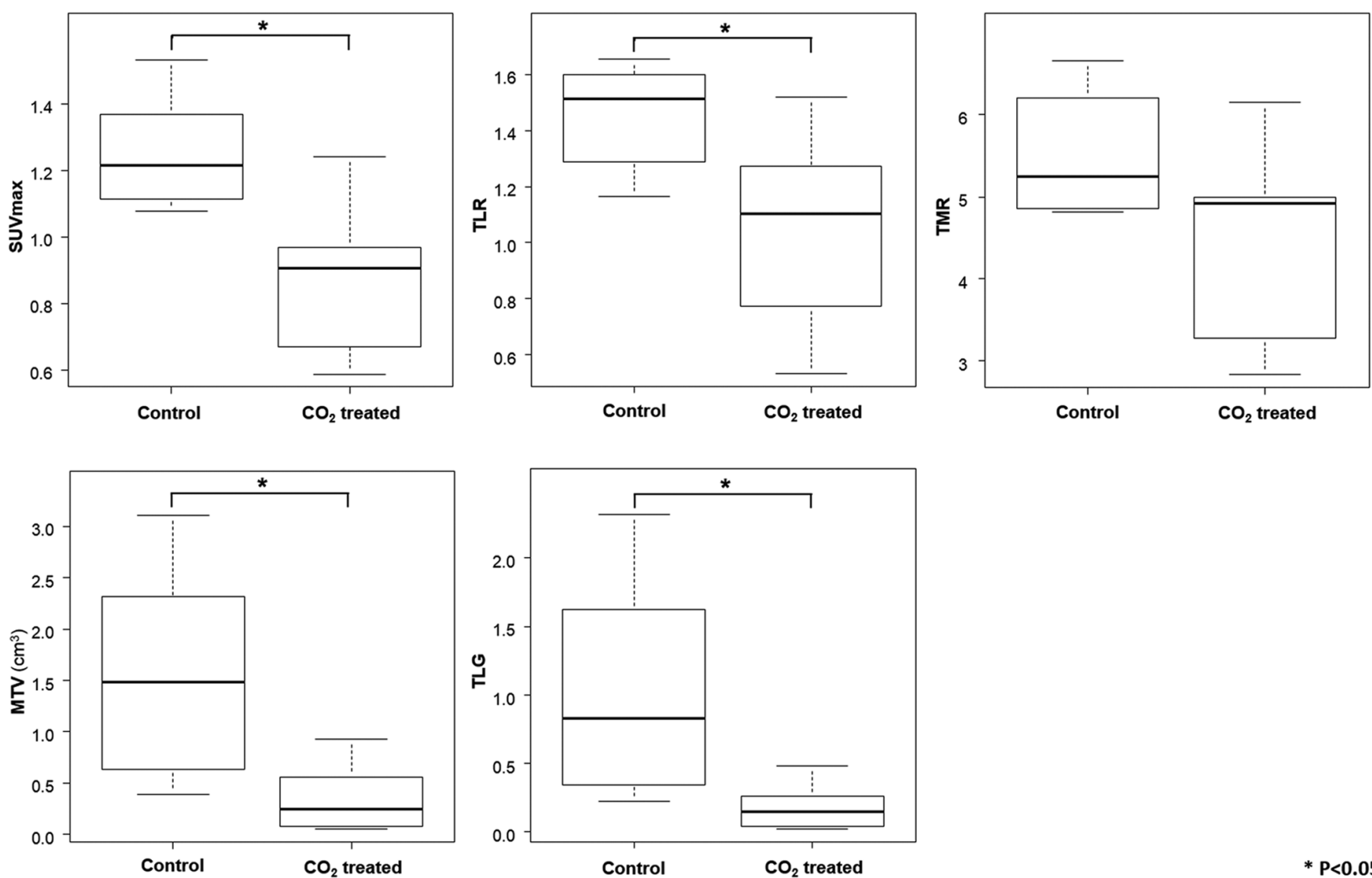

Figure 3. Box-and-whisker plots. The box-and-whisker plots demonstrate that the $\mathrm{SUV}_{\max }$, TLR, MTV and TLG are significantly lower in the $\mathrm{CO}_{2}$-treated group compared with the control group. There is no significant difference in the TLR between the two groups. $\mathrm{SUV}_{\max }$, maximum standardized uptake value; TLR, tumor-to-liver ratio; MTV, metabolic tumor volume; TLG, total lesion glycolysis.

than $10 \mathrm{mmHg}(22)$. Animal and human model studies have demonstrated a significant correlation between ${ }^{18} \mathrm{~F}-\mathrm{FMISO}$ uptake and the oxygen status of several tumors including gliomas, non-small cell lung cancer, head and neck cancer, cervical cancer, and rhabdomyosarcoma (12,14,23-25). Recently, the ${ }^{18} \mathrm{~F}$-FMISO uptake has been found to be associated with prognosis after treatment in certain tumors, such as in head and neck cancer $(26,27) .{ }^{18}$ F-FMISO PET-CT may be utilized not only for evaluation of the hypoxic state of the tumor before and after $\mathrm{CO}_{2}$ therapy, but also to predict the therapeutic effect.

In the present study, five quantitative parameters were used for the analysis of ${ }^{18} \mathrm{~F}$-FMISO PET-CT. PET images are usually converted to $\mathrm{SUV}_{\max }$, which is the most common conventional parameter. However, its use has some limitations, in that it is affected by many patient-associated and technical factors. Patient-associated factors include body size, body fat percentage, blood glucose level and tumor type, while technical factors include the signal-to-noise properties of PET scanners, accuracy of the image reconstruction algorithm, and time from administration to image acquisition. Without accounting for all these sources of error, the $\mathrm{SUV}_{\max }$ calculation could show $>50 \%$ error (28). Therefore, other incremental parameters are commonly used to evaluate PET imaging. The tumor-to-background ratio (TBR) is the ratio of $\mathrm{SUV}_{\max }$ of the tumor to the SUV of the non-tumoral area. A blood pool from the left ventricle and aorta were used as a background tissue in an ${ }^{18} \mathrm{~F}$-FMISO study (17). However, the left ventricle and aorta in mice are too small to set an accurate VOI; hence, the gluteal muscle and liver were used as a background tissue. No significant difference in TMR was found; this could have been due to the oxygenation of the gluteal muscles by percutaneous $\mathrm{CO}_{2}$ treatment. TLR was considered to be more reliable than TMR in measuring the therapeutic effect of $\mathrm{CO}_{2}$ in this study as $\mathrm{CO}_{2}$ was not administered in the liver.

In some studies, single-voxel-based parameters such as $\mathrm{SUV}_{\max }$ and TBR could not evaluate the entire tumor concentration, because most malignant tumors are heterogenous. The ${ }^{18} \mathrm{~F}$-FMISO concentration in this study was not homogeneous. This may reflect not only the heterogeneity of the cancer cell, but also the existence of necrotic areas. Volume-based parameters such as MTV and TLG may reveal overall tumor concentrations. Some studies reported that MTV and TLG were superior to single-voxel-based parameters in predicting treatment response in many tumors. In the present study, both, single-voxel-based and volume-based parameters decreased after $\mathrm{CO}_{2}$ treatment, indicating an improvement in the hypoxic condition of the tumor.

There are some limitations to the present study. Firstly, ${ }^{18} \mathrm{~F}$-FMISO was used, in which several hours are required after administration for obtaining the sufficient tumor/normal tissue ratio needed for visualization; due to its slow clearance and high nonspecific accumulation in normal tissues. Therefore, although the present study showed that $\mathrm{CO}_{2}$ treatment improved hypoxia, the temporal changes in intratumoral 
hypoxia after treatment, including the duration of $\mathrm{CO}_{2}$ action, were not evaluated in the present study. Dynamic PET studies or the use of newer tracers with higher clearance and greater accumulation in the hypoxic region may help overcome this limitation. Secondly, evaluation of true hypoxia is challenging in areas of poor blood flow due to difficulty in ${ }^{18} \mathrm{~F}-\mathrm{FMISO}$ infusion. Thirdly, bone tumor cells were transplanted subcutaneously, and the small animals used were different from the patients encountered in actual clinical practice. It may be necessary to conduct future research under conditions similar to those in actual clinical practice. Finally, no immunohistochemical assessment was conducted in this study; however, as mentioned, immunohistochemical assessment had already been previously performed using the same cell lines, and therefore were omitted from the present study.

In conclusion, ${ }^{18} \mathrm{~F}$-FMISO PET revealed that $\mathrm{CO}_{2}$ treatment improved intratumoral hypoxia in vivo. This technique enables assessment of the therapeutic effect by imaging and may contribute to the clinical application of $\mathrm{CO}_{2}$ treatment.

\section{Acknowledgements}

Not applicable.

\section{Funding}

This work was supported by JSPS KAKENHI (grant no. JP18K15547).

\section{Availability of data and materials}

The datasets used and/or analyzed during the current study are available from the corresponding author on reasonable request.

\section{Authors' contributions}

KM, TO, TU and KI conducted all experiments. HI, YK, KSa and TG contributed to the animal experiments. KM, EU, KSo, MN, MY and KSu contributed to data interpretation or data analysis. TO, YS, JH and TM conceived and designed this study. KM and TO wrote the manuscript. All authors contributed to the writing of the manuscript. All authors read and approved the final manuscript.

\section{Ethics approval and consent to participate}

The present study was approved by the Institutional Animal Care and Use Committees (approval nos. P160903 and 28-043-000) and performed in accordance with Animal Experimentation Regulations of Kobe University Graduate School of Medicine and Osaka University Graduate School of Medicine.

\section{Patient consent for publication}

Not applicable.

\section{Competing interests}

The authors declare that they have no competing interests.

\section{References}

1. Laking G and Price P: Radionuclide imaging of perfusion and hypoxia. Eur J Nucl Med Mol Imaging 37 (Suppl 1): S20-S29, 2010.

2. Vaupel P: The role of hypoxia-induced factors in tumor progression. Oncologist 9 (Suppl 5): 10-17, 2004.

3. Jensen FB: Red blood cell pH, the Bohr effect, and other oxygenation-linked phenomena in blood $\mathrm{O}_{2}$ and $\mathrm{CO}_{2}$ transport. Acta Physiol Scand 182: 215-227, 2004.

4. Mimeault M and Batra SK: Hypoxia-inducing factors as master regulators of stemness properties and altered metabolism of cancer- and metastasis-initiating cells. J Cell Mol Med 17: 30-54, 2013.

5. Hartmann BR, Bassenge E, Pittler $M$ and Hartmann BR: Effect of carbon dioxide-enriched water and fresh water on the cutaneous microcirculation and oxygen tension in the skin of the foot. Angiology 48: 337-343, 1997.

6. Liang J, Kang D, Wang Y, Yu Y, Fan J and Takashi E: Carbonate ion-enriched hot spring water promotes skin wound healing in nude rats. PLoS One 10: e0117106, 2015.

7. Sakai Y, Miwa M, Oe K, Ueha T, Koh A, Niikura T, Iwakura T, Lee SY, Tanaka M and Kurosaka M: A novel system for transcutaneous application of carbon dioxide causing an 'artificial Bohr effect' in the human body. PLoS One 6: e24137, 2011.

8. Onishi Y, Kawamoto T, Ueha T, Kishimoto K, Hara H, Fukase N, Toda M, Harada R, Minoda M, Sakai Y, et al: Transcutaneous application of carbon dioxide (CO2) induces mitochondrial apoptosis in human malignant fibrous histiocytoma in vivo. PLoS One 7: e49189, 2012.

9. Harada R, Kawamoto T, Ueha T, Minoda M, Toda M, Onishi Y, Fukase N, Hara H, Sakai Y, Miwa M, et al: Reoxygenation using a novel $\mathrm{CO}_{2}$ therapy decreases the metastatic potential of osteosarcoma cells. Exp Cell Res 319: 1988-1997, 2013.

10. Ueshima E, Yamaguchi M, Ueha T, Muradi A, Okada T, Idoguchi K, Sofue K, Akisue T, Miwa M, Fujii M, et al: Inhibition of growth in a rabbit VX2 thigh tumor model with intraarterial infusion of carbon dioxide-saturated solution. J Vasc Interv Radiol 25: 469-476, 2014.

11. Katayama N, Sugimoto K, Okada T, Ueha T, Sakai Y, Akiyoshi H, Mie K, Ueshima E, Sofue K, Koide Y, et al: Intra-arterially infused carbon dioxide-saturated solution for sensitizing the anticancer effect of cisplatin in a rabbit VX2 liver tumor model. Int J Oncol 51: 695-701, 2017.

12. Dubois L, Landuyt W, Haustermans K, Dupont P, Bormans G, Vermaelen P, Flamen P, Verbeken E and Mortelmans L: Evaluation of hypoxia in an experimental rat tumour model by [(18)F]fluoromisonidazole PET and immunohistochemistry. Br J Cancer 91: 1947-1954, 2004.

13. Sato J, Kitagawa Y, Yamazaki Y, Hata H, Okamoto S, Shiga T, Shindoh M, Kuge Y and Tamaki N: ${ }^{18} \mathrm{~F}$-fluoromisonidazole PET uptake is correlated with hypoxia-inducible factor-1 $\alpha$ expression in oral squamous cell carcinoma. J Nucl Med 54: 1060-1065, 2013.

14. Valk PE, Mathis CA, Prados MD, Gilbert JC and Budinger TF: Hypoxia in human gliomas: demonstration by PET with fluorine18-fluoromisonidazole. J Nucl Med 33: 2133-2137, 1992.

15. Onishi Y, Kawamoto T, Ueha T, Hara H, Fukase N, Toda M, Harada R, Sakai Y, Miwa M, Nishida K, et al: Transcutaneous application of carbon dioxide $\left(\mathrm{CO}_{2}\right)$ enhances chemosensitivity by reducing hypoxic conditions in human malignant fibrous histiocytoma. J Cancer Sci Ther 4: 7, 2012.

16. Watabe T, Kanai Y, Ikeda H, Horitsugi G, Matsunaga K, Kato H, Isohashi K, Abe K, Shimosegawa E and Hatazawa J: Quantitative evaluation of oxygen metabolism in the intratumoral hypoxia: ${ }^{18}$ F-fluoromisonidazole and 15O-labelled gases inhalation PET. EJNMMI Res 7: 16, 2017.

17. Koyasu S, Tsuji Y, Harada H, Nakamoto Y, Nobashi T, Kimura H, Sano K, Koizumi K, Hamaji M and Togashi K: Evaluation of tumor-associated stroma and its relationship with tumor hypoxia using dynamic contrast-enhanced CT and (18)F misonidazole PET in murine tumor models. Radiology 278: 734-741, 2016.

18. Hirata K, Kobayashi K, Wong KP, Manabe O, Surmak A, Tamaki $\mathrm{N}$ and Huang SC: A semi-automated technique determining the liver standardized uptake value reference for tumor delineation in FDG PET-CT. PLoS One 9: e105682, 2014.

19. Im HJ, Bradshaw T, Solaiyappan M and Cho SY: Current methods to define metabolic tumor volume in positron emission tomography: Which one is better? Nucl Med Mol Imaging 52: $5-15,2018$. 
20. Kanda Y: Investigation of the freely available easy-to-use software 'EZR' for medical statistics. Bone Marrow Transplant 48: 452-458, 2013.

21. Lee ST and Scott AM: Hypoxia positron emission tomography imaging with ${ }^{18} \mathrm{~F}$-fluoromisonidazole. Semin Nucl Med 37: 451-461, 2007.

22. Krohn KA, Link JM and Mason RP: Molecular imaging of hypoxia. J Nucl Med (Suppl 2) 49: 129S-148S, 2008.

23. Eschmann SM, Paulsen F, Reimold M, Dittmann H, Welz S, Reischl G, Machulla HJ and Bares R: Prognostic impact of hypoxia imaging with ${ }^{18} \mathrm{~F}$-misonidazole PET in non-small cell lung cancer and head and neck cancer before radiotherapy. J Nucl Med 46: 253-260, 2005.

24. Gagel B, Reinartz P, Demirel C, Kaiser HJ, Zimny M, Piroth M, Pinkawa M, Stanzel S, Asadpour B, Hamacher K, et al: [18F] fluoromisonidazole and [18F] fluorodeoxyglucose positron emission tomography in response evaluation after chemo-/radiotherapy of non-small-cell lung cancer: A feasibility study. BMC Cancer 6: 51, 2006.

25. Zimny M, Gagel B, DiMartino E, Hamacher K, Coenen HH, Westhofen M, Eble M, Buell U and Reinartz P: FDG - a marker of tumour hypoxia? A comparison with [18F]fluoromisonidazole and $\mathrm{pO}_{2}$-polarography in metastatic head and neck cancer. Eur J Nucl Med Mol Imaging 33: 1426-1431, 2006.
26. Kikuchi M, Yamane T, Shinohara S, Fujiwara K, Hori SY, Tona Y, Yamazaki H, Naito Y and Senda M: ${ }^{18} \mathrm{~F}$-fluoromisonidazole positron emission tomography before treatment is a predictor of radiotherapy outcome and survival prognosis in patients with head and neck squamous cell carcinoma. Ann Nucl Med 25: 625-633, 2011.

27. Asano A, Ueda S, Kuji I, Yamane T, Takeuchi H, Hirokawa E, Sugitani I, Shimada H, Hasebe T, Osaki A, et al: Intracellular hypoxia measured by ${ }^{18} \mathrm{~F}$-fluoromisonidazole positron emission tomography has prognostic impact in patients with estrogen receptor-positive breast cancer. Breast Cancer Res 20: 78, 2018.

28. Mah K and Caldwell CB: Biological target volume. In: PET-CT in Radiotherapy Treatment Planning. Paulino AC and Teh BS (eds). Content Repository Only, Philadelphia, pp52-89, 2008.

(i) (9) This work is licensed under a Creative Commons Attribution-NonCommercial-NoDerivatives 4.0 International (CC BY-NC-ND 4.0) License. 